\title{
Intégration du concept de la fiabilité en conception des prothèses orthodontiques: Application sur les systèmes de fixation par mini-plaques utilisées dans les mandibules fracturées
}

\section{Integration of reliability concept into orthodontic prosthesis design: Application on mini-plate fixation systems used in fractured mandibles}

\author{
Kharmanda G. ${ }^{1}$, Kharma M-Y. ${ }^{2,3}$, El-Hami A. ${ }^{4}$ \\ ${ }^{1}$ Département de Génie Biomédicale, Université de Lund, Lund, Suède, ghias.kharmanda@bme.Ith.se \\ ${ }^{2}$ Département de Chirurgie Maxillofacial, Collèges de Farabi, Jiddah, Arabie Saoudite, mykharma@hotmail.com \\ ${ }^{3}$ Département de Chirurgie Maxillofacial, Université d'Alep, Faculté de Médicine Dentaire, Syrie, mykharma@hotmail.com \\ ${ }^{4}$ Laboratoire d'Optimisation et Fiabilité en Mécanique des Structures, INSA de Rouen, abdelkhalak.elhami@insa-rouen.fr
}

RÉSUMÉ. Les prothèses orthodontiques peuvent être utilisées pour remplacer les dents (implants, ponts, couronnes ...) ou pour reconstruire les dents (rétraction ...) ou les fractures (systèmes de fixation ...). Dans ce travail, on s'intéresse aux systèmes de fixation surtout la fixation des fractures mandibulaires par mini-plaques. Une simulation numérique de la mandibule fracturée est réalisée pour identifier le mode (le scenario) de défaillance. Ensuite, une analyse de fiabilité est effectuée pour évaluer la stabilisation des systèmes de fixation utilisés pour les fractures de mandibule.

ABSTRACT. Orthodontic prostheses can be used to replace teeth (dental implants, bridges, crowns ...) or reconstruct teeth (retraction ...) or fractures (fixation systems ...). In this work, we are interested in fixation systems especially the fixation of mandible fractures using mini-plates. A numerical simulation of a fractured mandible is carried out in order to identify the failure mode (or scenario). Next a reliability analysis is carried out in order to evaluate the stabilization of the fixation systems for the mandible fractures.

MOTS-CLÉS. Fiabilité structurale, Conception de prothèses orthodontiques, Mandibule fracturée, Fixation par mini-plaques. KEYWORDS. Structural Reliability, Orthodontic Prosthesis Design, Mandible Fractures, Mini-plate Fixation.

\section{Introduction}

Les recherches en biomécanique utilisent des approches en modélisation géométrique, mécanique et une analyse expérimentale proches de la mécanique des structures et de matériaux. Cependant, de nombreux verrous existent en amont et en aval pour caractériser et personnaliser la géométrie, les matériaux dont le comportement varie en fonction des lois de remodelage encore mal connues, ainsi que les chargements mécaniques. L'intégration des concepts de fiabilité a pour objectif de considérer l'incertitude sur plusieurs aspects dont le chargement et les propriétés des matériaux de l'os. Dans ce papier, l'analyse de fiabilité en prothèses orthodontiques a été effectuée pour évaluer la stabilisation après une acte chirurgicale. La stratégie de la fixation par mini-plaques est utilisée pour le traitement des mandibules fracturées. Durant l'opération chirurgicale, les muscles peuvent être abimée où coupées. Pour cela, l'incertitude est appliquée sur le chargement dans ce travail en considérant la force de morsure et les forces des muscles comme variables aléatoires. Une application de l'analyse de fiabilité est faite sur la mandibule fracturée en considérant une structure d'un matériau homogène isotrope pour évaluer la stabilisation des systèmes de fixation utilisés pour les fractures de mandibule en comparant avec les observations du chirurgien. 


\section{Matériaux et méthodes}

\subsection{Description de Problème}

La reconstitution 3D de la géométrie de la mandibule fracturée traitée avec les mini-plaques et les vis a été effectuée en utilisant le logiciel SOLIDWORK. Les vis sont considérées comme des cylindres pour simplifier la modélisation géométrique (figure 1a). Les deux parties de la mandibule fracturée sont composées d'un matériau homogène de l'os cortical avec un comportement isotrope où le module de Young égale à $14 \mathrm{GPa}$ et le coefficient de Poisson égale à 0.3 . Pour calculer la limite d'élasticité de l'os, on utilise la formulation optimisée entre la limite d'élasticité et le module de Young [KHA 15], ce qui conduit à une limite d'élasticité de l'os égale à 112.35MPa. Les min-plaques et les vis sont en titane avec un module de Young $110 \mathrm{GPa}$ et un coefficient de Poisson 0.34 [KOR 07]. La limite d'élasticité des mini-plaques et des vis égale à $860 \mathrm{MPa}$. Pour le modèle du maillage sur ANSYS, le nombre des éléments finis utilisés est : 14845. Les éléments utilisés sont non-linéaires de types : CONTACT \& SOLID. Le contact entre les surfaces de la fracture est considérée non-pénétré avec une distance initiale de $100 \mu \mathrm{m}$. Le contact entre les mini-plaques et les vis est totalement bondé. Les forces des muscles inclues dans le modèle étudié sont masséter superficiel (Superficial Masseter: SM), masséter profonde (Deep Masseter: DM), temporal antérieur (Anterior Temporalis: AT), temporal médial (Medial Temporalis: MT) temporal postérieur (Posterior Temporalis: PT) et ptérygoïdien médial (Medial Pterygoid: MP). Pendant le processus de morsure, les muscles digastriques ne sont pas significativement actives et alors, on peut les ignorer dans l'analyse pour simplifier l'étude [RAM 14]. Selon la figure 1b, la force de morsure est appliquée en région A. Les sommes de forces de muscles de masséter sont notées $M^{D}$ (droit) et $M^{G}$ (gauche) sont appliquées en régions B et C. Les sommes de forces de muscles de temporal sont notées $T^{D}$ (droit) et $T^{G}$ (gauche) sont appliquées en régions D et E. Finalement, Les sommes des forces de muscles de ptérygoïdien sont notées $P^{D}$ (droit) et $P^{G}$ (gauche) sont appliquées en régions $\mathrm{F}$ et $\mathrm{G}$. La mandibule est serrée en régions $\mathrm{H}$ et $\mathrm{I}$.

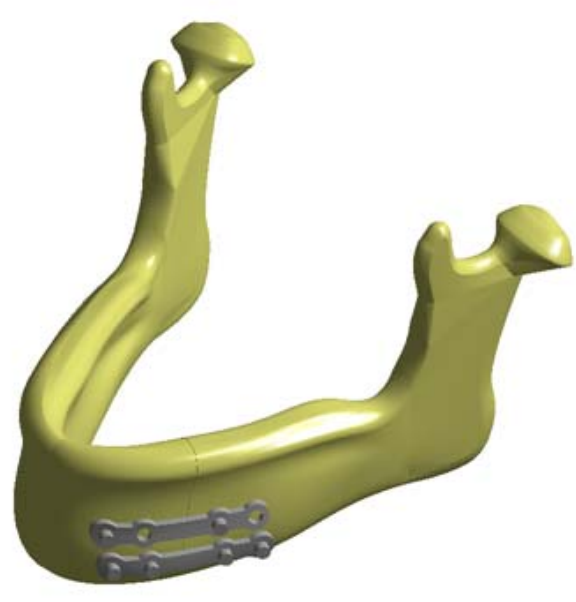

$a$

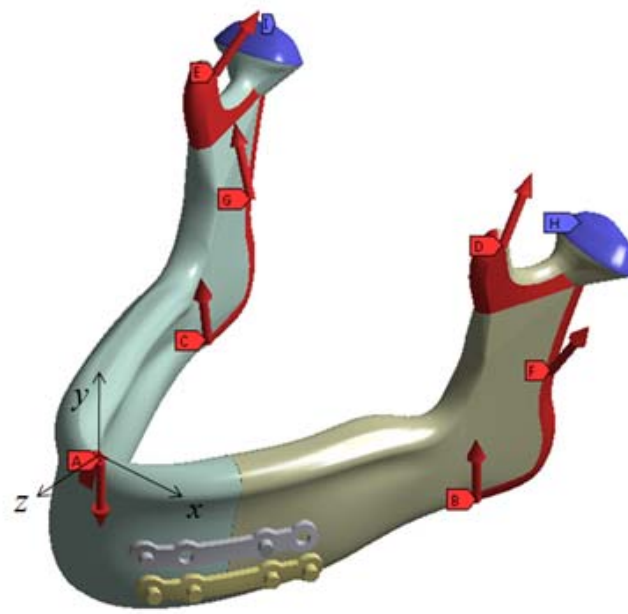

$b$

Figure 2.1. a) : Modèle géométrique de la mandibule fracturée avec les mini-plaques et les vis, b) Conditions aux limites

Après l'opération chirurgicale, le patient doit alimenter par des repas doux pour avoir une valeur faible de force de morsure. Selon les résultats expérimentaux de Kumar et al. [KUM 13], après la chirurgie, la valeur maximale de la force de morsure postopératoire est $F=44 \mathrm{~N}$. Les efforts des muscles présentés dans le tableau 1, sont mesurés à la capacité maximale d'une mandibule non fracturée [MES 11], ce qui correspond à une force de morsure de $F=208 N$. 


\begin{tabular}{|c|c|c|c|}
\hline Muscle Forces & $\boldsymbol{F}_{\boldsymbol{x}}[\mathbf{N}]$ & $\boldsymbol{F}_{\boldsymbol{y}}[\mathbf{N}]$ & $\boldsymbol{F}_{\boldsymbol{z}}[\mathbf{N}]$ \\
\hline Superficial Masseter (SM) & 18.2 & 303.3 & 12.1 \\
\hline Deep Masseter (DM) & 7.8 & 128.3 & 15.6 \\
\hline Anterior Temporalis (AT) & -18.4 & 104.8 & -43.8 \\
\hline Medial Temporalis (MT) & -6.5 & 36.3 & -53.1 \\
\hline Posterior Temporalis (PT) & -3.4 & 6.8 & -37 \\
\hline Medial Pterygoid (MP) & 187.4 & 325.1 & -76.5 \\
\hline
\end{tabular}

Tableau 2.1. Forces des muscles pour une mandibule non-fracturée [MES 11]

En conséquence, dans le modèle numérique, les forces utilisées sont proportionnelles d'une façon que la force de morsure est : $F=44 \mathrm{~N}$. On considère ces valeurs comme les valeurs moyennes de notre problème. De plus, la prise en compte de l'incertitude sur les forces permet de prendre en compte un intervalle de confiance puisqu'il existe plusieurs forces actives inconnues.

\subsection{Analyse de fiabilité}

Pour l'étude de fiabilité, trois scenarios de défaillance peuvent avoir lieu. Mathématiquement, on peut écrire les contraintes sous la forme suivante :

$$
\begin{aligned}
& H_{1}=\delta_{\max }-\delta_{w} \leq 0 \\
& H_{2}=\sigma_{\max }^{\text {Metal }}-\sigma_{y}^{\text {Metal }} \leq 0 \\
& H_{3}=\sigma_{\max }^{\text {Os }}-\sigma_{y}^{\text {Os }} \leq 0
\end{aligned}
$$

Le déplacement relatif maximal dans la zone de la fracture ne doit pas dépassé une valeur requise $\left(H_{1}=\delta_{\max }-\delta_{w} \leq 0\right)$ et la valeur maximale de la contrainte de von-Mises effective dans les mini-plaques $\left(H_{2}=\sigma_{\max }^{\text {Metal }}-\sigma_{y}^{\text {Metal }} \leq 0\right)$ et également dans l'os $\left(H_{3}=\sigma_{\max }^{\text {Os }}-\sigma_{y}^{O s} \leq 0\right)$. Le problème de fiabilité peut être écrit sous la forme suivante :

$$
\begin{aligned}
\min : & d\left(u_{i}\right)=\sqrt{\sum_{i=1}^{n} u_{i}^{2}} \\
\text { sous }: H_{1}\left(u_{i}\right) & =\delta_{\max }\left(u_{i}\right)-\delta_{w} \leq 0 \\
: & H_{2}\left(u_{i}\right)=\sigma_{\max }^{\text {Metal }}\left(u_{i}\right)-\sigma_{y}^{M} \leq 0 \\
: H_{3}\left(u_{i}\right) & =\sigma_{\max }^{\text {Os }}\left(u_{i}\right)-\sigma_{y}^{M} \leq 0
\end{aligned}
$$

où $u_{i}$ est le vecteur des variables normées suivantes la loi normale (Gaussienne). Le but de trouver le MPP qui appartient à l'état limite $\max \left(H_{1}\left(u_{i}\right), H_{2}\left(u_{i}\right), H_{3}\left(u_{i}\right)\right)=0$. Le point MPP se trouve lorsque la distance entre l'origine de l'espace normé et l'état limite est minimale [KHA 10]. L'algorithme de fiabilité développé a pour le but de trouver le point de défaillance le plus probable (MPP: Most Probable failure Point). Les variables aléatoires de ces forces sont 19 composantes. Dans ce cas-la, on a 18 forces de muscles et 1 force de morsure. Les écart-types sont proportionnels aux valeurs moyennes de $50 \%$.

\subsection{Résultats numériques}

Après une simulation sur ANSYS, on obtient des réponses correspondantes aux valeurs moyennes où l'état de cette structure est dans le domaine de sûreté. La figure 2 a montre la distance entre les deux surfaces de la mandibule fracturée $\delta$. 


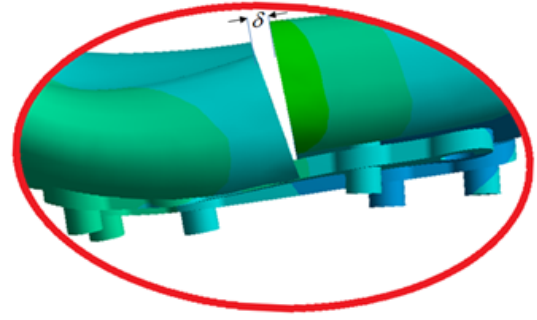

$\boldsymbol{a}$

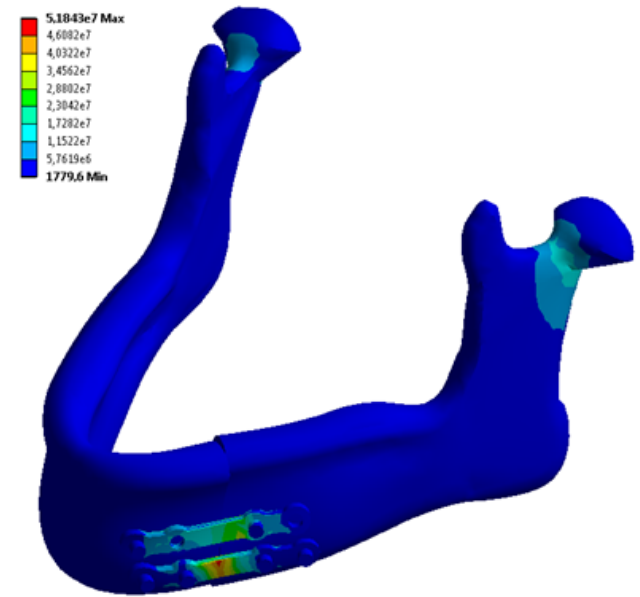

b

Figure 2.2. a) Déplacement relative, b) Distribution de contraintes de von-Mises

Les résultats dans le tableau 2 montre qu'il n'y a plus de symétrie par rapport au plan de l'origine $x=0$. La force du coté gauche $M_{y}^{G}$ est significativement moins que celles du coté droit $M_{y}^{D}$ au point MPP. La force de morsure est presque doublée par rapport à sa moyenne $F \approx 82 \mathrm{~N}$.

\begin{tabular}{|c|c|c|c|}
\hline \multicolumn{2}{|c|}{ Paramètres } & Moyennes & MPP \\
\hline $\boldsymbol{F}[\mathbf{N}]$ & $F_{y}$ & -44 & -81.66 \\
\hline \multirow[b]{2}{*}{$M^{D}[\mathrm{~N}]$} & $M_{x}^{D}$ & 5.46 & 1.47 \\
\hline & $M_{y}^{D}$ & 90.64 & 57.48 \\
\hline \multirow{4}{*}{$M^{G}[\mathrm{~N}]$} & $M_{z}^{D}$ & 5.82 & 10.64 \\
\hline & $M_{x}^{G}$ & -5.46 & -5.56 \\
\hline & $M_{y}^{G}$ & 90.64 & 41.50 \\
\hline & $M_{z}^{G}$ & 5.82 & 9.51 \\
\hline \multirow{3}{*}{$T^{D}[\mathrm{~N}]$} & $T_{x}^{D}$ & -5.94 & -6.84 \\
\hline & $T_{y}^{D}$ & 31.06 & 18.00 \\
\hline & $T_{z}^{D}$ & -28.12 & -51.10 \\
\hline \multirow{3}{*}{$T^{G t}[\mathrm{~N}]$} & $T_{x}^{G}$ & 5.94 & 9.45 \\
\hline & $T_{y}^{G}$ & 31.06 & 46.42 \\
\hline & $T_{z}^{G}$ & -28.12 & -20.57 \\
\hline \multirow{4}{*}{$P^{D}[\mathrm{~N}]$} & $P_{x}^{D}$ & 39.35 & 45.44 \\
\hline & $P_{y}^{D}$ & 68.27 & 75.48 \\
\hline & $P_{z}^{D}$ & -16.07 & -15.74 \\
\hline & $P_{x}^{G}$ & -39.35 & -42.66 \\
\hline \multirow[t]{2}{*}{$P^{G}[\mathrm{~N}]$} & $P_{y}^{G}$ & 68.27 & 56.88 \\
\hline & $P_{z}^{G}$ & -16.07 & -19.13 \\
\hline
\end{tabular}

Tableau 2.2. Valeurs moyennes et le point de défaillance (MPP)

Le tableau 3 montre les contraintes maximales de von-Mises des différentes composantes. Au point des valeurs moyennes, la valeur maximale de von-Mises est de 52MPa et se trouve à la plaque inférieure (figure 1b). 


\begin{tabular}{|c|c|c|c|}
\hline Etats limites & Paramètres & Moyennes & MPP \\
\hline $\boldsymbol{G}_{\mathbf{1}}$ & $\delta_{\max }(\mu \mathrm{m})$ & 131 & 149.1 \\
\hline $\boldsymbol{G}_{\mathbf{2}}$ & $\sigma_{\max }^{\text {PlaqueSup }}(\mathrm{MPa})$ & 51.84 & 65.04 \\
\hline $\boldsymbol{G}_{\mathbf{2}}$ & $\sigma_{\max }^{\text {PlaueInf }}(\mathrm{MPa})$ & 33.51 & 48.83 \\
\hline $\boldsymbol{G}_{\mathbf{3}}$ & $\sigma_{\max }^{G}(\mathrm{MPa})$ & 19.07 & 26.07 \\
\hline $\boldsymbol{G}_{\mathbf{3}}$ & $\sigma_{\max }^{D}(\mathrm{MPa})$ & 17.78 & 29.34 \\
\hline & $\beta$ & --- & 4.17 \\
\hline & $P_{f}$ & --- & $1.6 \times 10^{-5}$ \\
\hline
\end{tabular}

Tableau 2.3. Valeurs de paramètres de sortie

\section{Discussion et conclusion}

$\mathrm{Au}$ point MPP, la contrainte maximale de von-Mises égale à $65 \mathrm{MPa}$, ce qui indique qu'il existe un grand marge par rapport à la rupture de mini-plaques. La contrainte maximale de von-Mises pour les valeurs moyennes des forces égale à $19 \mathrm{MPa}$. Lorsque le point MPP est trouvé, la contrainte maximale de von-Mises dans l'os égale à $29 \mathrm{MPa}$, ce qui indique aussi qu'il existe une marge par rapport aux conditions choisies au niveau de contraintes. Cependant, le problème se trouve dans le déplacement relative à la surface de fracture où sa valeur à la première simulation est : $\delta_{\text {max }}=131 \mu \mathrm{m}$. Pour le point MPP, on considère que l'état limite $\delta_{\max }-\delta_{w}=0$ doit être satisfait. Pour la condition de guérison, le déplacement relative ne doit pas dépasser une certaine valeur $\left(\delta_{\max }=149.1 \approx \delta_{w}=150 \mu m\right)$ [COX 03]. En considérant la variabilité des forces, l'indice de fiabilité égale à $\beta=4.17 \in[3-4.25]$, ce qui correspond à une probabilité de défaillance $\left(P_{f} \approx 1.6 \times 10^{-5}\right)$. L'importance de l'intégration de l'analyse de fiabilité dans ce cas-là est d'évaluer la stabilité de la structure après l'opération chirurgicale.

\section{Bibliographie}

[COX 03] COX T., KoHN M.W., IMPELluso T., « Computerized analysis of resorbable polymer plates and screws for the rigid fixation of mandibular angle fractures », Journal of Oral and Maxillofacial Surgery, vol. 61, p. 481-487, 2003.

[KHA 10a] KHARMANDA G., El-HAMI A., SOUZA DE CURSI E., « Reliability-Based Design Optimization», In edited book Multidisciplinary Design Optimization in Computational Mechanics, Edited by Piotr Breitkopt and Rajan Filomeno Coelho, Chapter 11, : Wiley \& Sons, April 2010, ISBN: 9781848211384, Hardback, vol. 576, pp (2010).

[KHA 15] KHARMANDA G., «Reliability analysis for cementless hip prosthesis using a new optimized formulation of yield stress against elasticity modulus relationship », Materials and Design, vol. 65, p. 496-504, 2015.

[KOR 07] KORKMAZ H.H., « Evaluation of different mini-plates in fixation of fractured human mandible with the finite element method », Oral Surgery, Oral Medicine, Oral Pathology and Oral Radiology, vol. 103, e1-e13, 2007.

[KUM 13] KUMAR S.T., SARAF S., DEVI S.P., « Evaluation of Bite Force After Open Reduction and Internal Fixation Using Microplates », Journal of Dentistry, Tehran University of Medical Sciences, vol. 10, n 5, p. 466-477, 2013.

[MES 11] Mesnard M., Ramos A., Ballu A., Morlier J. Cid M., Simoes J.A., « Biomechanical analysis comparing natural and alloplastic temporomandibular joint replacement using a finite element model », Journal of Oral and Maxillofacial Surgery, vol. 69, n 4, p. 1008-1017, 2011.

[RAM 14] Ramos A., MARQues H., MESnard M., " The effect of mechanical properties of bone in the mandible, a numerical case study », Advances in Biomechanics and Applications, vol. 1, n 1, p. 067-076, 2014. 